\title{
Effects of process parameters on dimensional precision and tensile strength of wax patterns for investment casting by selective laser sintering
}

\author{
Qian Wei, Run-nan Wang, *Qing-yan Xu, Bai-cheng Liu \\ Key Laboratory for Advanced Materials Processing Technology (MOE), School of Materials Science and Engineering, Tsinghua University, \\ Beijing 100084, China
}

\begin{abstract}
Turbine blades, produced by the directional solidification (DS) process, often require high dimensional accuracy and excellent mechanical properties. A critical step in their production is the fabrication of wax patterns. However, the traditional manufacturing process has many disadvantages, such as long-term production, low material utilization rate, and the high cost of producing a complex-shaped wax pattern. Selective laser sintering (SLS) is one of the most extensively used additive manufacturing techniques that substantially shortens the production cycle. In this study, SLS was adopted to fabricate the wax pattern instead of the traditional manufacturing process. The orthogonal experiment method was carried out to investigate the effects of laser power, scanning speed, scanning space, and layer thickness on the dimensional precision and morphologies of the SLS parts. The SLS parts showed a minimum dimensional deviation when laser power, scanning speed, scanning space, and layer thickness were $10 \mathrm{~W}$, $3000 \mathrm{~mm} \cdot \mathrm{s}^{-1}, 0.18 \mathrm{~mm}$, and $0.25 \mathrm{~mm}$, respectively. In addition, the tensile strength and fracture morphologies were closely associated with the laser volumetric energy density (VED). The tensile strength reached a maximum when the VED was $0.0762 \mathrm{~J} \cdot \mathrm{mm}^{-3}$, with an evident brittle fracture morphology. The wax pattern manufactured in this way meets the accuracy and strength requirements for investment casting. This research offers a novel path for the production of wax patterns for complex-shaped turbine blades by SLS.
\end{abstract}

Key words: polystyrene; additive manufacturing; SLS; investment casting; orthogonal experiment

CLC numbers: TG222 Document code: A

Article ID: 1672-6421(2018 04-299-08

$\mathrm{T}$

he wax patterns of single-crystal superalloy turbine

blade, with the necessary complex shapes and high dimensional accuracy, are mainly manufactured by waxinjectors. Traditional wax pattern materials are paraffinstearin, paraffin-polyolefin, and modified rosin, etc. The traditional wax pattern is prepared using the wax injector, followed by the dipping in the ceramic slurry, stuccoing, and drying, then a ceramic shell mould is obtained. The quality of the turbine blades depends heavily on this series of processes. Fabricating the wax pattern is the first step in the investment casting process, and its processing quality is transmitted to the ceramic shell and then to the final casting ${ }^{[1-2]}$. Accordingly, the quality of the wax pattern plays a significant role in the entire casting process, which is a time-consuming and laborious work. Furthermore, the quality of the

\section{*Qing-yan Xu}

Male, born in 1971, Ph.D, Professor. Research interests: materials processing engineering, modeling and simulation of directional solidification process for superalloy turbine blade.

E-mail: scjxqy@tsinghua.edu.cn

Received: 2018-02-01; Accepted: 2018-03-20 wax pattern is significantly constrained by the wax injector machine. At present, the problems with wax patterns produced by domestic wax injectors are often associated with dimensional instability, misrun, bubbles, surface roughness, deformation, and adhesion, greatly impairing the formability of the castings. Therefore, the development of a new technology for controlling wax defects is required.

Selective laser sintering (SLS) is an innovative technique for producing a casting's wax pattern, comparable to conventional manufacturing methods. SLS is free from the limitation of moulds for conventional methods, and hence is superior in the manufacture of wax patterns with complex shapes. SLS uses a laser beam as its energy source. Figure 1 is a schematic of the temperature distribution of the laser. The laser beam reaches the fabrication platform, and a temperature field is formed around the laser beam. The temperature field presents a Gaussian distribution. The laser energy melts the individual and irregular polystyrene (PS) powders, and forms the sintered necks.

Efforts ${ }^{[3-7]}$ have been made to investigate the optimal process parameters for producing sintered parts by 


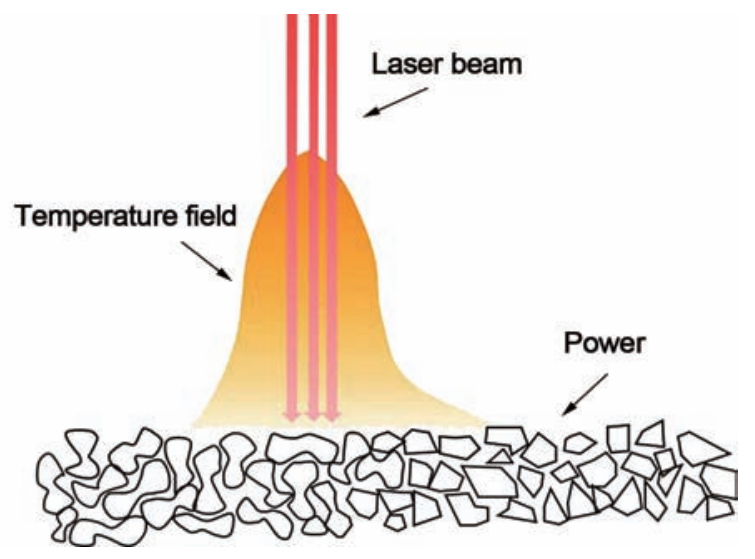

Fig. 1: Schematic of temperature distribution of laser

SLS. It has been acknowledged that process parameters have a significant effect on the sintering quality of SLS parts, while multiple-factor coupling effects have rarely been taken into account. There have also been many research studies carried out to explain the effect of energy density on sintered parts. $\mathrm{HO} \mathrm{H}$. CH. et al. ${ }^{[8]}$ studied the effect of energy density on the morphology and properties of selective laser-sintered parts, and found that the high energy beam enabled compacted components to be obtained. However, when the energy density was extremely high, degradation of the polymer occurred. Pavan et al. ${ }^{[9]}$ estimated the thermo-temporal effect influenced by the energy density and the inter-layer time, on the resulting SLS PA12 part quality at both the micro-level and macro-level, but ignored the influence of layer thickness. In recent years, with the ever-growing development of computer software, many researchers have applied numerical analysis to simulate the sintering processes and microscopic evolution ${ }^{[10-14]}$. Ganeriwala et al. ${ }^{[12]}$ employed a discrete element model to model the deposition and heat transfer involved in the SLS process at a very small scale, but without considering thermal interaction with the surrounding atmosphere. Peyre et al. ${ }^{[15]}$ combined experimental design with numerical simulation to estimate the thermal cycles and resulting fusion depths of PA12 and PEKK polymers. However, they did not consider multifactor coupling. Most studies are confined to the application of sintered parts, and few studies have combined the technical study of SLS parts with engineering applications ${ }^{[16-18]}$, remaining at the stage of theoretical research. Furthermore, the dimensional precision and strength of polystyrene parts do not satisfy the engineering requirements, which is unacceptable for wax patterns. Additionally, the relationship between the optimum design of processing parameters and the morphological evolution law of sintered parts has not been explicitly identified. Consequently, there is a pressing need to research the problems consistent with practical engineering applications.

Considering the strong demand for turbine blade wax patterns, this study aims to realize the fast production of wax patterns with sufficient strength. A wax pattern for a turbine blade for investment casting was fabricated by SLS. First, the orthogonal experiment design ${ }^{[19]}$ was adopted to explore the optimum process parameters in terms of dimensional precision and the range analysis was applied to analyze the experimental results. Then, tensile strength and fracture morphologies of SLS samples with different laser volumetric energy densities (VED) were analyzed. Finally, the wax pattern for a turbine blade with a complex surface were manufactured using the comprehensive optimal process parameters. The future prospect of SLS technology was also discussed.

\section{Experimental procedure}

\subsection{Materials}

PS has a low specific heat, proper melt viscosity, favorable fluidity and thermal stability, which is favorable to the sintering formability of SLS parts. Its average particle size is $65 \mu \mathrm{m}$ with irregular particle shapes detected by a scanning electron microscope (ZEISS, Merlin Compact, Germany) (Fig. 2). Its glass transition temperature is $108{ }^{\circ} \mathrm{C}$, which determines the processing temperature of the SLS process, as detected by a synchronized thermal analyzer (DSC STA449F3, NETZSCH, Germany); the curves of PS powder can be seen in Fig. 3.

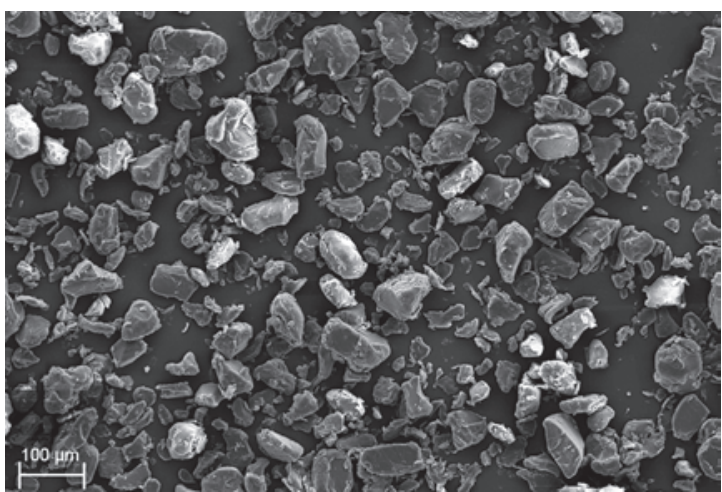

Fig. 2: Morphology of PS powder

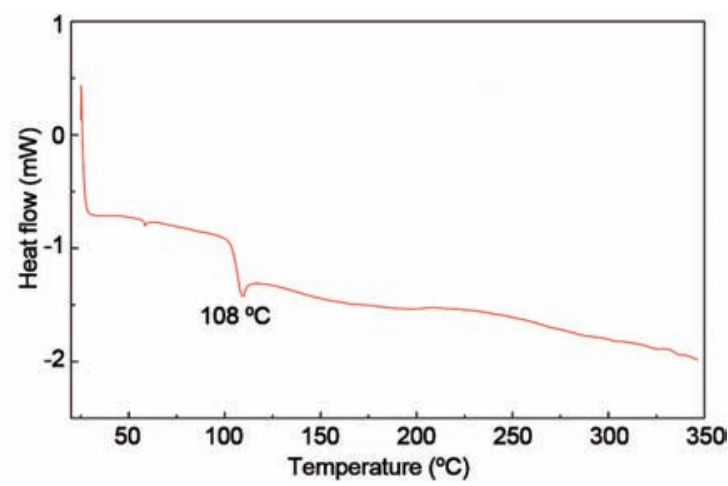

Fig. 3: DSC curves of PS powder

\subsection{Preparation of SLS samples}

The experiments were performed in a laser forming machine (HK S320, Huake 3D Technology Co. Ltd., China). The laser power was continuously adjustable within $55 \mathrm{~W}$; the laser wavelength, forming precision, maximum scanning speed, and maximum forming space were $10.6 \mu \mathrm{m}, \pm 0.2 / 200 \mathrm{~mm}, 4000 \mathrm{~mm} \cdot \mathrm{s}^{-1}$, and $320 \mathrm{~mm} \times 320 \mathrm{~mm} \times 450 \mathrm{~mm}$, respectively. According to the DSC curves of PS powder (Fig. 3), $88^{\circ} \mathrm{C}$ was chosen as the preheating temperature for all the experiments. The orthogonal experiments 
were employed to optimize the coupling process parameters. The design of the four factors and three levels $\left[\mathrm{L}_{9}\left(3^{4}\right)\right]$ of the orthogonal experiments is shown in Table 1.

In order to ensure that the properties of each group of samples were consistent, twenty samples of each group were arranged in parallel and formed at the same time. Figure 4 shows the main dimensions of the tensile samples.

Table 1: Four factors and three levels of orthogonal experiments

\begin{tabular}{|c|c|c|c|c|}
\hline \multirow{2}{*}{ Level } & \multicolumn{4}{|c|}{ Factors } \\
\hline & $\begin{array}{c}\text { A } \\
\text { Laser power (W) }\end{array}$ & $\begin{array}{c}\text { B } \\
\text { Scanning speed }\left(\mathrm{mm} \cdot \mathrm{s}^{-1}\right)\end{array}$ & $\begin{array}{c}\text { C } \\
\text { Scanning space }(\mathrm{mm})\end{array}$ & $\begin{array}{c}\text { D } \\
\text { Layer thickness }(\mathrm{mm})\end{array}$ \\
\hline 1 & 8 & 3,000 & 0.18 & 0.15 \\
\hline 2 & 10 & 3,500 & 0.20 & 0.20 \\
\hline 3 & 12 & 4,000 & 0.22 & 0.25 \\
\hline
\end{tabular}

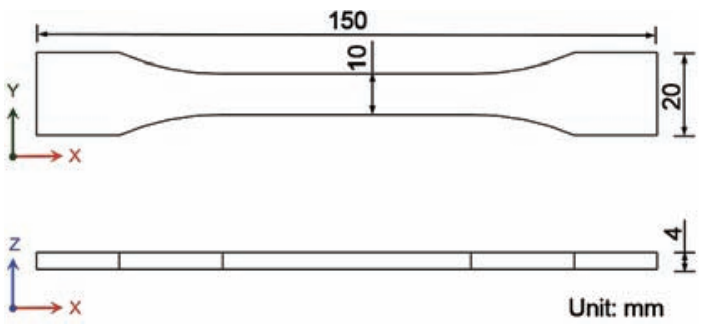

Fig. 4: Main dimensions of tensile samples

\subsection{Property tests}

(i) Dimensional measurements: After laser sintering, the surface of the PS parts was often covered with unnecessary powder. Samples with a relatively good surface quality could be obtained using a powder-purifying tool. A digital vernier caliper (0-150 mm, Guanglu, China) was used to measure the dimensions of the tensile samples, and the average dimensions of each group of twenty samples were calculated and used to characterize the dimensional deviation in different directions.

(ii) Tensile test: An electronic universal testing machine (ZWICKZ020, ZWICK Ltd., Germany) was used to conduct the tensile tests, according to the ASTM standard D638-14 ${ }^{[20]}$. All samples were tested at quasi-static and low strain rate conditions. The tensile rate was $2 \mathrm{~mm} \bullet \mathrm{s}^{-1}$. At least 15 samples of each group were adopted to conduct the tensile tests to minimize errors.

(iii) Scanning electron microscopy (SEM) observation: A low voltage was chosen to minimize heat accumulation and heat damage to the samples, and all samples were sputter coated with gold to eliminate the charged effect and to enhance the conductivity of samples. The samples were then observed under SEM (Merlin Compact, ZEISS, Germany) to evaluate their fracture surface morphologies.

\section{Results and discussion}

\subsection{Average dimension deviation of SLS parts}

Through an orthogonal experiment design, the coupling effects of laser power, scanning speed, scanning space, and layer thickness on the X-direction average deviation $(\Delta L)$ and Y-direction average deviation $(\Delta W)$ of the SLS-sintered parts were calculated, as shown in Fig. 5. As previously observed by researchers ${ }^{[3]}$, the SLS process parameters have different effects on the shrinkage of the SLS specimens. It was found that the average deviation in dimensions fluctuated between $0.196-0.732 \mathrm{~mm}$ for all samples. S4 is the most desirable orthogonal combination group, showing the smallest deviation with the theoretical 3D model, with $0.196 \pm 0.085 \mathrm{~mm}$ in $\Delta L$ and $0.228 \pm 0.044 \mathrm{~mm}$ in $\Delta W$.

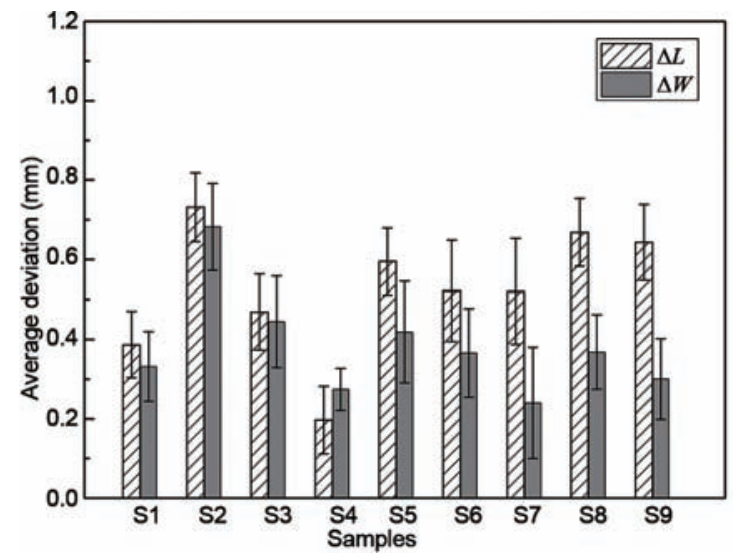

Fig. 5: Average deviation of each group of SLS samples

It can be seen that the average deviations in the X-direction $(\Delta L)$ are relatively higher than those in the Y-direction $(\Delta W)$ for all samples, which is mainly caused by the laser scanning mode, whereby a laser rotating mirror scans the focused laser beam into a linear facula. The scanning mode adopted in this experiment is vector-scanning mode; there is a scan interval between two adjacent parallel scanning lines along the $\mathrm{X}$-axis, and the existence of these intervals causes less energy to be exerted on the material particles, leading to a larger dimensional deviation in the $\mathrm{X}$-direction. In addition, the $\mathrm{X}$-direction dimension of the part is much larger than the Y-direction dimension, and hence, the effect of error accumulation is much greater in the X-direction. As a result, the average deviation in the $\mathrm{X}$-direction has been chosen as the evaluating indicator.

Table 2 lists the results of the orthogonal experiments. In order to determine the influence degree of different processing parameters, the range analysis has been adopted. From Fig. 5 , the average deviation in X-direction dimension $(\Delta L)$ of the 
samples is relatively larger than the Y-direction $(\Delta W)$, which means $\Delta L$ is more sensitive than $\Delta W$ to processing parameters. For this reason, the range analysis has been discussed based on $\Delta L$. Table 3 is the range analysis results of $\Delta L$.

$$
\begin{gathered}
\omega_{i j}=\frac{K_{i j}}{3}-\bar{x}_{i} \\
R_{j}=\max \left(\omega_{1 j}, \omega_{2 j}, \omega_{3 j}\right)-\min \left(\omega_{1 j}, \omega_{2 j}, \omega_{3 j}\right)
\end{gathered}
$$

where $K_{i j}$ is the sum of the test indicators corresponding to the $i$ level of the $j$ factor $(i=1,2,3, j=\mathrm{A}, \mathrm{B}, \mathrm{C}, \mathrm{D}) . \omega_{i j}$ is the difference between $K_{i j} / 3$ and the mean value. $R_{j}$ reflects the variation amplitude of the experimental index when the level of the $j$ column factor fluctuates. The larger the value of $j$, the greater the influence on the experimental index.

\begin{tabular}{|c|c|c|c|c|c|c|}
\hline \multirow{2}{*}{ Samples } & \multirow{2}{*}{$\begin{array}{l}\text { Laser power (A) } \\
\text { (W) }\end{array}$} & \multirow{2}{*}{$\begin{array}{l}\text { Scanning speed (B) } \\
\qquad\left(\mathrm{mm} \cdot \mathrm{s}^{-1}\right)\end{array}$} & \multirow{2}{*}{$\begin{array}{l}\text { Scanning space (C) } \\
\qquad(\mathrm{mm})\end{array}$} & \multirow{2}{*}{$\begin{array}{c}\text { Layer thickness (D) } \\
\text { (mm) }\end{array}$} & \multicolumn{2}{|c|}{ Average deviation } \\
\hline & & & & & $\Delta L(\mathrm{~mm})$ & $\Delta W(\mathrm{~mm})$ \\
\hline S1 & 8 & 3,000 & 0.18 & 0.15 & $0.386 \pm 0.083$ & $0.276 \pm 0.073$ \\
\hline S2 & 8 & 3,500 & 0.20 & 0.20 & $0.731 \pm 0.087$ & $0.568 \pm 0.091$ \\
\hline S3 & 8 & 4,000 & 0.22 & 0.25 & $0.468 \pm 0.095$ & $0.370 \pm 0.096$ \\
\hline S4 & 10 & 3,000 & 0.20 & 0.25 & $0.196 \pm 0.085$ & $0.228 \pm 0.044$ \\
\hline S5 & 10 & 3,500 & 0.22 & 0.15 & $0.595 \pm 0.084$ & $0.348 \pm 0.106$ \\
\hline S6 & 10 & 4,000 & 0.18 & 0.20 & $0.521 \pm 0.127$ & $0.305 \pm 0.093$ \\
\hline S7 & 12 & 3,000 & 0.22 & 0.20 & $0.520 \pm 0.133$ & $0.200 \pm 0.117$ \\
\hline S8 & 12 & 3,500 & 0.18 & 0.25 & $0.668 \pm 0.085$ & $0.306 \pm 0.078$ \\
\hline S9 & 12 & 4,000 & 0.20 & 0.15 & $0.671 \pm 0.095$ & $0.250 \pm 0.065$ \\
\hline
\end{tabular}

Table 2: Results of orthogonal experiments

Table 3: Range analysis results of $\Delta L$

$\begin{array}{ccccc}\text { Indicators } & \begin{array}{c}\text { Laser power (A) } \\ (\mathbf{W})\end{array} & \begin{array}{c}\text { Scanning speed }(\mathbf{B}) \\ \left(\mathrm{mm}^{-1}\right)\end{array} & \begin{array}{c}\text { Scanning space (C) } \\ (\mathbf{m m})\end{array} & \begin{array}{c}\text { Layer thickness }(\mathbf{D}) \\ (\mathrm{mm})\end{array} \\ K_{1 j} & 1.585 & 1.103 & 1.576 & 1.653 \\ K_{2 j} & 1.313 & 1.995 & 1.600 & 1.773 \\ K_{3 j} & 1.859 & 1.662 & 1.583 & 1.333 \\ \omega_{1 j} & 0.000 & -0.161 & -0.003 & 0.022 \\ \omega_{2 j} & -0.091 & 0.136 & 0.005 & 0.062 \\ \omega_{3 j} & 0.091 & 0.025 & -0.001 & -0.084 \\ R_{j} & 0.182 & 0.297 & 0.008 & 0.147 \\ \text { Factors } & & \text { Important B>A>D>C Secondary } & \end{array}$

As the $K_{i j}$ value is required to be as small as possible while judging dimensional precision, the optimal combination of process parameters is $\mathrm{A}_{2} \mathrm{~B}_{1} \mathrm{C}_{1} \mathrm{D}_{3}$, i.e., the laser power, scanning speed, scanning space, and layer thickness are $10 \mathrm{~W}, 3000$ $\mathrm{mm} \cdot \mathrm{s}^{-1}, 0.18 \mathrm{~mm}$, and $0.25 \mathrm{~mm}$, respectively. According to the calculation of $R_{j}$, the order in which the parameters most significantly affect the dimensional deviation of samples are B (scanning speed), followed by A (laser power), D (layer thickness), and C (scanning space).

To further investigate the effect of different ranges of each process parameter on the dimensional accuracy of the samples, the curves of average dimensional deviation vs. each factor are shown in Fig. 6. With an increase in laser power, the average dimensional deviation of the SLS samples firstly decreases and then increases, while the other three process parameters present an opposite trend. As the laser power increases, the laser energy density increases, and heat-affected area broadens, so the binding force between layers is strengthened ${ }^{[3]}$, and discrete PS particles begin to melt and consolidate into a whole, which leads to a small average deviation of the samples. However, the 
PS particles gasify and lose their bonding capability under a higher laser power, increasing the average deviation of samples [Fig. 6(a)]. Scanning speed also affects the distribution of laser energy and heat; as scanning speed increases from 3,000 $\mathrm{mm} \cdot \mathrm{s}^{-1}$ to $4,000 \mathrm{~mm} \cdot \mathrm{s}^{-1}$, the laser energy and heat per unit time per unit area decreases, which leads to insufficient melting of the PS particles, causing a great average deviation. When the scanning speed exceeds a certain value, the average deviation in $\Delta L$ decreases, this may be due to the fact that the binding force between the particles is strengthened, and leads to a small dimensional deviation, as shown in Fig. 6 (b). The scanning space is the distance between two adjacent parallel scan lines, which influences the laser energy per unit area. Scanning space is the least influential factor among the four process parameters, with the smallest average deviation changes of the samples. The average deviation in $\Delta L$ first increases and then decreases
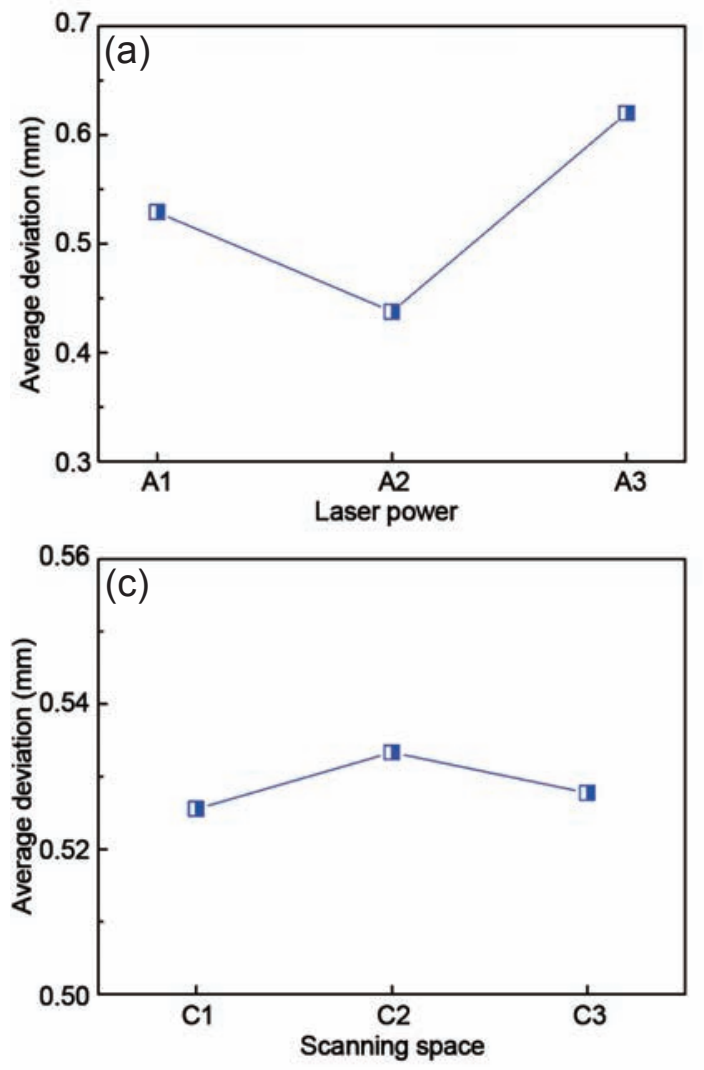

Fig. 6: Average deviation of dimensions depending on single factors

\subsection{Tensile strength}

All samples were tested at quasi-static and high strain rate conditions. For all the tensile samples, fractures occurred between the gauge length of samples, indicating that the experimental data obtained was valid.

In order to better characterize the morphology of sintered parts, an approach has been proposed by Ciurana et al. ${ }^{[21]}$ in which the VED is used to characterize the sintering quality. The definition of VED is shown in Eq. (3).

$$
V E D=\frac{P}{v \sigma t}
$$

where $P$ is the laser power (W), $v$ is the scanning speed $\left(\mathrm{mm} \bullet \mathrm{s}^{-1}\right), \sigma$ is the scanning space $(\mathrm{mm})$, and $t$ is the layer thickness $(\mathrm{mm})$. with increasing scanning space [Fig. 6(c)]. This is because with a smaller scanning space, the particles melt more due to the overlapping scan lines. With an increasing scanning space, the laser energy and heat accumulation cannot adequately maintain the sintering process, and cause a large average deviation of the samples. The layer thickness is the vertical distance between two neighboring layers, and is closely associated with the laser energy per unit volume. As layer thickness increases from 0.15 $\mathrm{mm}$ to $0.20 \mathrm{~mm}$, the strong binding force between the layers weakens, leading to a large deviation. As the layer thickness increases to $0.25 \mathrm{~mm}$, the laser energy distribution per unit time per unit volume is more uniform in an extreme threedimensional micro zone, causing a small deviation [Fig. 6(d)]. It is also recommended that the process parameters should be in accordance with the properties of materials and the characteristics of the equipment used.
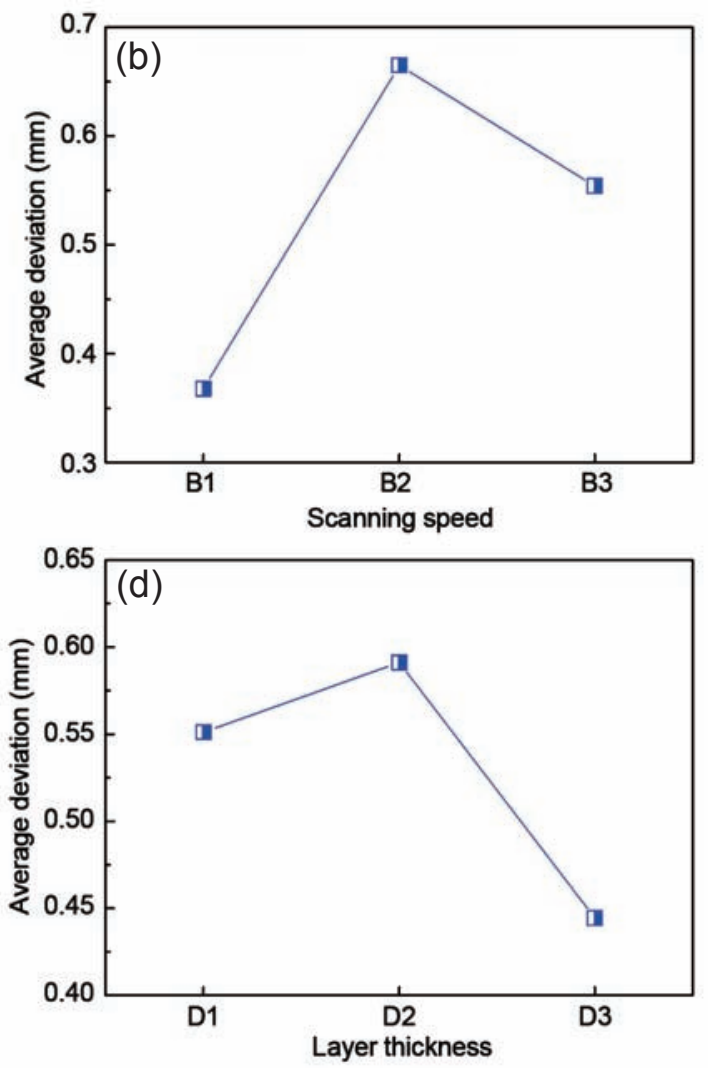

The changes of VED and tensile strength of samples are shown in Fig. 7. The tensile strength of the SLS parts increases at the beginning, and then decreases after reaching the maximum as the VED increases. The powder is not in a viscous state at a low VED, which causes poor fluidity between particles; the particles are simply stacked together, leading to a low tensile strength. With an increase in VED, the powder changes from partially melted to totally melted, the connection between particles strengthens, and at $0.0762 \mathrm{~J} \bullet \mathrm{mm}^{-3}$, the tensile strength reaches a maximum. When the VED is too high, the melt partially gasifies which causes many pores to present in the samples, so the strong connections weaken, resulting in the decrease of tensile strength. 


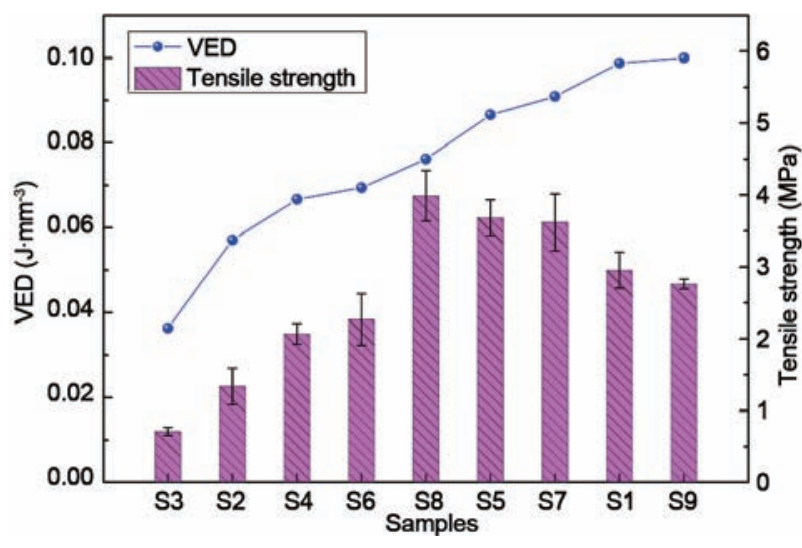

Fig. 7: VED and tensile strengths of samples

\subsection{Morphology analysis}

For all the tensile samples, the fracture zones occurred in the gauge length of samples, indicating that a normal rupture mode could be obtained. Figure 8 shows the evolution of the fracture morphologies of nine groups of samples under different VEDs. The particle shape of S3 sample, sintered at the minimum
VED, is irregular angular [Fig. 8(a)], with a shape similar to the raw PS powder. The individual particles can be identified, demonstrating that the energy input is barely sufficient to melt the powder, so the particles are poorly connected or even unbonded. The morphology remains unchanged until the VED

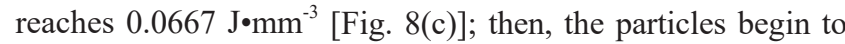
melt and gradually join into a whole body, forming a sintered neck between two connected particles. As the VED increases from $0.0667 \mathrm{~J} \bullet \mathrm{mm}^{-3}$ to $0.1000 \mathrm{~J} \bullet \mathrm{mm}^{-3}$ [Fig. 8(c)-(i)], it can be seen that the fractures all occur in the sintered necks. The average size of the cross-sectional area of the sintered neck increases and then decreases as the VED increases, and reaches the maximum at $\mathrm{VED}=0.0762 \mathrm{~J} \cdot \mathrm{mm}^{-3}$, which is in accordance with the tensile strength. The cracks easily propagate between the sintered necks, leaving small fracture sections. When the energy density is too high $\left[\mathrm{VED}=0.100 \mathrm{~J} \mathrm{~mm}^{-3}\right.$, Fig. 8(i)], the size of the cross-sectional area decreases sharply and the number of pores increases. As a result, the internal structure is less dense, which greatly worsens the tensile strength of the SLS-sintered parts. This is consistent with the findings of Ho H.C.H. ${ }^{[8]}$.
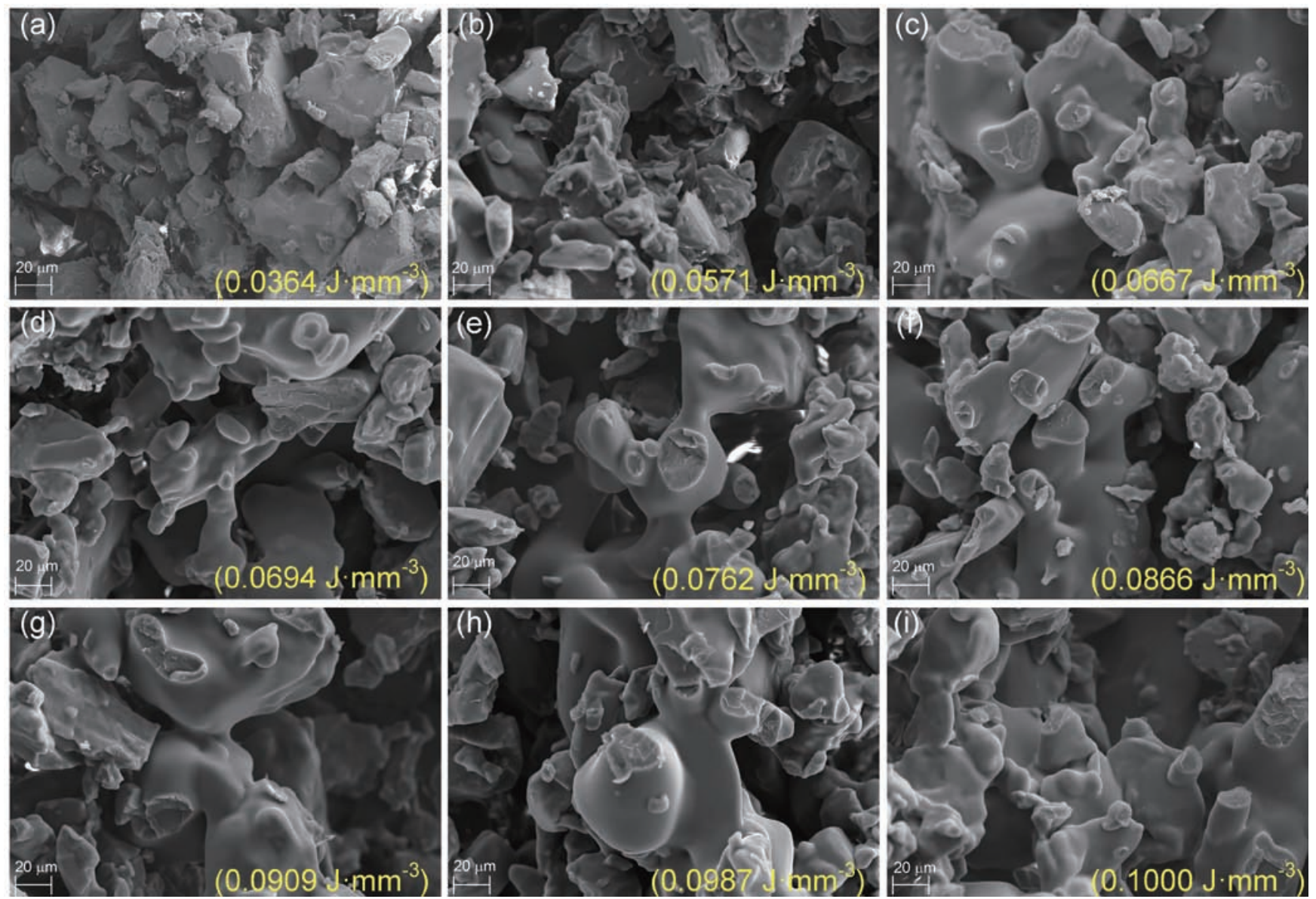

Fig. 8: Fracture surface morphologies of tensile samples under different VEDs: (a) S3; (b) S2; (c) S4; (d) S6; (e) S8; (f) S5; (g) S7; (h) S1; (i) S9

Figure 9 is a magnified view of the cross section fracture of sample S8. It shows a river pattern - a typical brittle fracture morphology, in which tributaries intersect and then form a larger step, and many of these steps then merge forming the river pattern. Along the direction of fracture, many small steps converge into large steps and then into even larger steps, from the "upstream" to the "downstream" of the river. The flow of the river coincides with the direction of crack propagation, hence, using the flow of the river pattern, the expansion direction of the cleavage crack and crack source in the microscopic area could be estimated. 


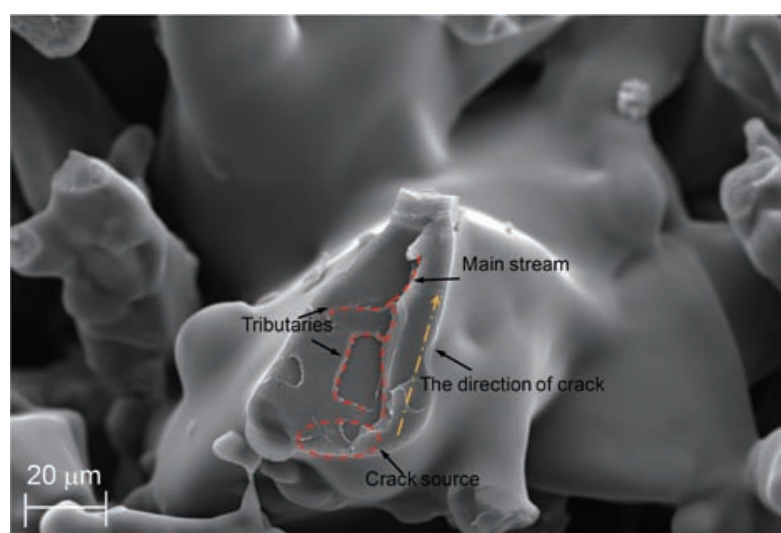

Fig. 9: Magnified view of cross section fracture of S8
The optimal process parameters based on minimum dimensional deviation were adopted to fabricate the turbine blade wax pattern. Figure 10 shows the final SLS part and the 3D dimensional deviation of the turbine blade wax pattern. The wax pattern perfectly retained the thin-wall structure and had a good surface quality [Fig. 10(a)]. The 3D deviation cloud diagram of the wax pattern shows that the average dimensional deviation is within the range of -0.114 to $0.150 \mathrm{~mm}$, which guarantees the dimensional precision and dimensional stability of the wax pattern [Fig. 10(b)]. When combined with traditional shell making technology, a ceramic shell mould with high quality can be obtained rapidly and effectively.
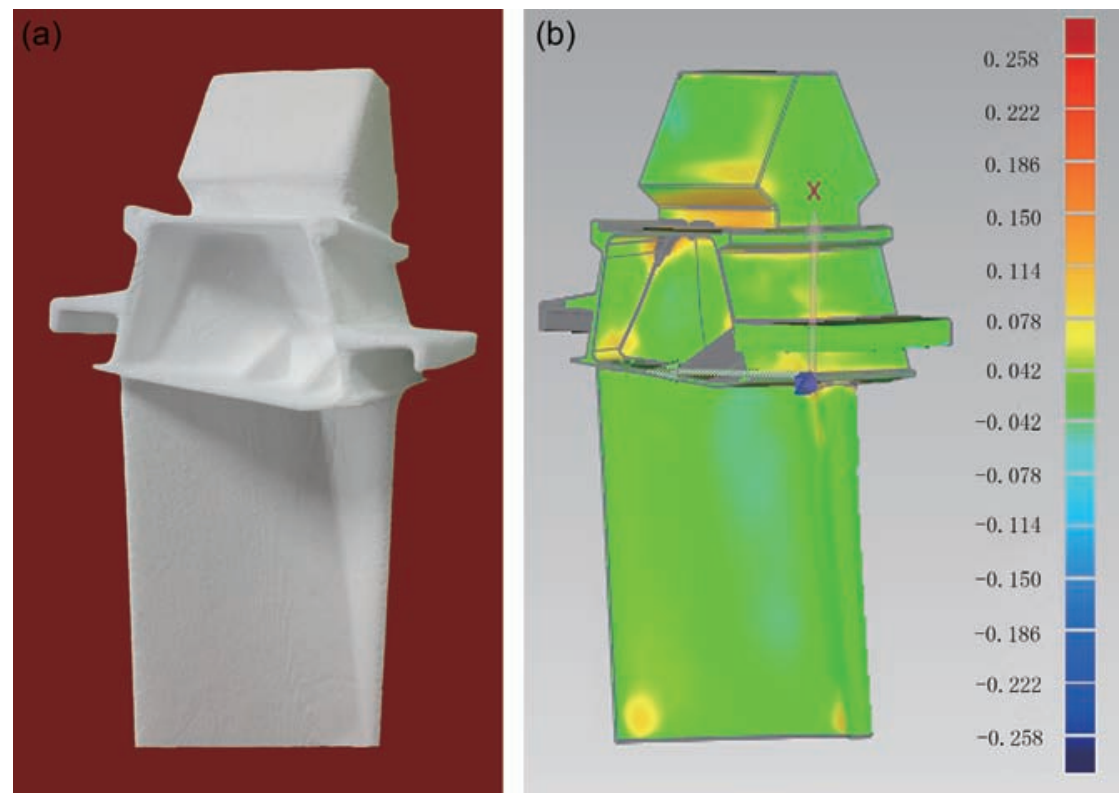

Fig. 10: Turbine blade wax pattern: (a) final SLS part; (b) 3D dimensional deviation compared with theoretical model

\section{Conclusions}

(1) SLS method was used for fabricating investment casting wax patterns. Laser power, scanning speed, scanning space, and layer thickness exert different influences on the dimensional precision, tensile strength, and fracture morphology of the parts produced by SLS. The SLS-sintered PS patterns have the optimum dimensional accuracy when laser power, scanning speed, scanning space, and layer thickness are $10 \mathrm{~W}, 3000 \mathrm{~mm} \bullet \mathrm{s}^{-1}, 0.18 \mathrm{~mm}$, and $0.25 \mathrm{~mm}$, respectively.

(2) The tensile strength is closely associated with the laser volumetric energy density (VED), and reaches a maximum when the VED is $0.0762 \mathrm{~J} \cdot \mathrm{mm}^{-3}$.

(3) The fracture morphology of the SLS parts exhibits evident brittle fracture characteristics. Different VEDs have different effects on the microstructures of the investigated parts. A rational VED is required to obtain desirable comprehensive properties of the parts, corresponding with the optimal processing parameters.

(4) By combining this technique with traditional shell-making technology, the fabrication of a wax pattern of a turbine blade for investment casting in a fast and effective way can be realized.

\section{References}

[1] Pradyumna R, Sridhar S, Satyanarayana A, et al. Wax patterns for integrally cast rotors/stators of aeroengine gas turbines. Materials Today: Proceedings, 2015, 2(4): 1714-1722.

[2] Taşcıoğlu S, Akar N. A novel alternative to the additives in investment casting pattern wax compositions. Materials \& Design, 2003, 24(8): 693-698.

[3] Liu K, Shi Y S, Li C H, et al. Indirect selective laser sintering of epoxy resin- $\mathrm{Al}_{2} \mathrm{O}_{3}$ ceramic powders combined with cold isostatic pressing. Ceramics International, 2014, 40(5): 7099-7106.

[4] Zheng H Z, Zhang J, Lu S Q. Effect of core-shell composite particles on the sintering behavior and properties of nano- $-\mathrm{Al}_{2} \mathrm{O}_{3} l$ polystyrene composite prepared by SLS. Materials Letters, 2006(60): 1219-1223.

[5] Athreya S R, Kalaitzidou K, Das S. Processing and characterization of a carbon black-filled electrically conductive Nylon-12 nanocomposite produced by selective laser sintering. Materials Science and Engineering: A, 2010, 527(10): 26372642.

[6] Wang R J, Wang L L, Zhao L H. Influence of process parameters on part shrinkage in SLS. International Journal of Advanced Manufacturing Technology, 2007, 5-6(33): 498-504.

[7] Strano G, Hao L, Everson R, et al. Multi-objective optimization 
of selective laser sintering processes for surface quality and energy saving. Proceedings of the Institution of Mechanical Engineers, Part B: Journal of Engineering Manufacture, 2011, 9(225): 1673-1682.

[8] Ho H C H, Gibson I, Cheung W L. Effects of energy density on morphology and properties of selective laser sintered polycarbonate. Journal of Materials Processing Technology, 1999, 89-90: 204-210.

[9] Pavan M, Faes $M$, Strobbe $D$, et al. On the influence of interlayer time and energy density on selected critical-to-quality properties of PA12 parts produced via laser sintering. Polymer Testing, 2017, 61: 386-395.

[10] Childs T H C, Berzins M, Ryder G R, et al. Selective laser sintering of an amorphous polymer-simulations and experiments. Proceedings of the Institution of Mechanical Engineers Part B Journal of Engineering Manufacture, 1999, 4(213): 333-349.

[11] He W T, Wei Q S, Liu K, et al. Numerical simulation of cold isostatic pressed alumina parts produced by selective laser sintering and part shape optimization. Ceramics International, 2013, 39(8): 9683-9690.

[12] Ganeriwala R, Zohdi T I. Multiphysics modeling and simulation of selective laser sintering manufacturing processes. Procedia CIRP, 2014, 14: 299-304

[13] Wang Z J, He W T, Shi Y S, et al. Experimental based full process simulation of alumina selective laser processed parts densified by cold isostatic pressing and solid state sintering. Journal of the European Ceramic Society, 2014, 34(7): 1853-1863.
[14] Kolossov S, Boillat E, Glardon R, et al. 3D FE simulation for temperature evolution in the selective laser sintering process. International Journal of Machine Tools and Manufacture, 2004, 44(2): 117-123.

[15] Peyre P, Rouchausse $Y$, Defauchy D, et al. Experimental and numerical analysis of the selective laser sintering (SLS) of PA12 and PEKK semi-crystalline polymers. Journal of Materials Processing Technology, 2015, 225: 326-336.

[16] Yang J S, Shi Y S, Shen Q W, et al. Selective laser sintering of HIPS and investment casting technology. Journal of Materials Processing Technology, 2009, 209(4): 1901-1908.

[17] Freyer F, Wirtz H, Freyer $\mathrm{C}$. Investment casting shell in 1 day using selective laser sintering (SLS). Foundryman, 2000, 1(93): 63-65.

[18] Yan M X, Zhou C, Tian X Y, et al. Design and selective laser sintering of complex porous polyamide mould for pressure slip casting. Materials \& Design, 2016, 111: 198-205.

[19] Dey A. Orthogonal fractional factorial designs. New York: Wiley, 1985.

[20] Standard Test Method for Tensile Properties of Plastics. ASTM D638-14, 2015.

[21] Ciurana J, Hernandez L, Delgado J. Energy density analysis on single tracks formed by selective laser melting with CoCrMo powder material. The International Journal of Advanced Manufacturing Technology, 2013, 68(5-8): 1103-1110.

This work was financially supported by National Science and Technology Major Project (2017ZX04014001-002), China-EU (European Union) Science \& Technology Cooperation in Aviation, Horizon 2020 Framework Programme for Research and Innovation (2014-2020) of EU (No. 690725) as well as National Natural Science Foundation of China (No. 51374137). 